\title{
Game theoretic approach for fertilizer application: looking for the propensity to cooperate
}

\author{
S. Schreider, P. Zeephongsekul and B. Abbasi \\ School of Mathematical and Geospatial Sciences, RMIT University, Melbourne, Victoria, Australia
}

Email: Sergei.Schreider@rmit.edu.au

\begin{abstract}
Application of game theoretic methods in resource management has become quite a well developed area over the last decades. An extended review of papers devoted to this aspect of resource management can be found in Schreider et al. (2010). Among the papers cited there, two important contributions to cooperative game application to resource management should be mentioned, and these are the papers by Filar and Gaertner (1997) where cooperative game is used in modeling greenhouse gases, and Dinar (2004) where a cooperative approach is used to understand the decision making process and its consequences on a drainage basin. Many researchers stress the importance of cooperation and apply the cooperative game theory in order to treat the problem of optimal distribution of limited resources. In our opinion, the two most important research questions in these areas are firstly, what mathematical models are most suitable in describing the problems and, secondly, how realistic are the mechanisms which are available to enable stakeholder to realize this cooperation in practice. In the present paper, the problem of finding an optimal fertilizer application using game theoretic approach to the Hopkins River catchment, Western Victoria, is considered. The players (agents) are local farmers who apply a certain amount of fertilizers in order to increase household revenue, but this also has a negative effect since it tends to increase the amount of phosphorus exported from paddocks to downstream waterways. Therefore, the relevant strategies selected by farmers are the quantities of phosphorus which should be applied to their lands. The amount of phosphorus applied by each player also defines the payoff or objective function for the player. Each payoff function contains three components. The first is the positive revenue obtained from the crop and the second is the negative component characterizing the dollar value spent on purchasing fertilizers. The damage caused by the blue green algae blooming in the catchment, which is also expressed in dollar value, is a third negative component in the payoff function. The model proposed in the previous paper (Schreider et al. 2010) treats solutions of the game in a noncooperative way (as a Nash equilibrium) and as a set of Pareto optima which characterize the semi-cooperative equilibria in the sense that players made a binding agreement not to deviate from their agreed choice of actions which would lead to better outcomes to all players. In Section 1, we provide the game theoretic formulation of phosphorus pollution of waterways which was first expounded in Schreider et al. (2010) where the formulation of the two notions of equilibria were discussed. Section 2 looks at using cooperative game theory based on formation of coalitions to model fertilizer application and presents a characteristic function for this game. We then give a brief discussion of the Shapley values for all players in the game. In Section 3, we apply the preceding results to the Hopkins River case study and a detailed comparison between the noncooperative and cooperative solutions to the case study will be given in Section 4. Finally the paper is concluded in Section 5 with a summary of the major outcomes of this study and suggestions for further research.
\end{abstract}

Keywords: Water quality, Phosphorus application, Cooperative games, Shapley values 


\section{GAME THEORETIC FORMULATION OF PHOSPHORUS POLLUTION OF WATERWAYS}

The game theoretic formulation for the problem considered in this paper was provided in full extend in Schreider et al. (2010). The important thing, which should be reminded here, is that the strategies for each player (farmer) are defined as an amount of Phosphorus he/she introduced annually on his/her property. Then the utility function was defined as a combined farmer's revenue constituted from crop production revenue and (negative) value spend for purchasing fertilisers. The crop revenue was calculated as a production times market prices computed for each crop produced by the player, where the production were calculated using the Cobb-Douglass production function of two production factors: water and fertiliser (Phosphorus). The utility function also has an environmental component, computed as a negative downstream impact explained by use of Phosphorus by the players. The negative environmental impact selected for this study is blue-green algae blooms due to phosphorus exported from the farmlands into the river catchment system. Thirty farms $(n=30)$ were chosen from the region and the three farming enterprise were wool, prime lamb (P lamb) and regular crops (crop) comprising wheat, oats and canola. All these enterprises benefitted from application of phosphorus. The reader is directed to Schreider et.al., (2010), for a detailed discussion on how these parameters were estimated and on the rationale behind them. The columns 2 and 3 of table 1 display the Nash equilibrium and Pareto optimum solutions for phosphorus application ( $\mathrm{kg} / \mathrm{ha}$ ) with $\gamma=0.115$ for later comparisons. Among the plethora of possible Pareto solutions, we have selected the one using equal weights since intuitively, this is the Pareto optimum that would maximize the sum of utilities.

\section{COOPERATIVE GAME THEORY BASED ON COALITION FORMATION}

In Section 1, we investigated the Hopkins catchment pollution problem, i.e. the blue-green algae blooms caused by phosphorus exported from farms into the surrounding river system, as a non-cooperative game involving several farmers. The game's objective was to optimize the payoff given by (2) when an individual player is pitted against all other players. The solutions to the non-cooperative game are the combinations of strategies by each player which satisfy Nash equilibrium, or Pareto optimum. On the other hand, the formation of coalitions and guaranteed payoff to each coalition are the main components in the theory of cooperative game based on coalition formation. The key problem considered in these games is not necessarily on how to obtain the optimal strategies but rather on how to distribute the payoff among members of a coalition so that no member would feel compelled to leave the coalition. Note here that we assume that the payoff is a transferable utility, i.e. it can be added and subtracted in the usual separable way. We next define the characteristic function $\nu(K): 2^{N} \longmapsto R$, where $2^{N}$ is the power set of $N$, associated with a cooperative game. A characteristic function is a real valued function defined over the set of all possible coalitions and forms a vital ingredient of a cooperative game. The value $\nu(K)$ can be interpreted as the joint utility e.g. worth, power, income, which the coalition $K$ is guaranteed if its members act cooperatively together as a group. Characteristic functions must satisfy

$$
\nu(\emptyset)=0 \quad \text { and } \quad \nu(A \cup B) \geq \nu(A)+\nu(B)
$$

for any mutually exclusive coalitions $A$ and $B$. The second condition stated that a larger coalition will be guaranteed an equal or larger payoff than the sum of individual payoffs in case the players splintered into smaller coalitions. This property is commonly referred to as the superadditivity property. In a coalitional game, the payoffs to the players are represented by the vector $\mathbf{x}=\left(x_{1}, x_{2}, \ldots, x_{n}\right)$. An admissible $\mathbf{x}$ which satisfies the following two conditions is known as an imputation under the characteristic function $\nu($.$) :$

$$
\begin{aligned}
& x_{i} \geq \nu(\{i\}) \text { foralli; } \\
& \sum_{i=1}^{n} x_{i}=\nu(N) .
\end{aligned}
$$

The first condition is the condition of individual rationality and is a reasonable assumption to make; otherwise, each player will not be induced to join a coalition. The second condition is called the condition of group rationality. To see that it is reasonable assumption, assume that $\sum_{i=1}^{n} x_{i}<\nu(N)$. Then the 
group can achieve $\nu(N)$ together and share it between themselves in such a way that each can receive an amount $\bar{x}_{i}>x_{i}$. On the other hand, if $\sum_{i=1}^{n} x_{i}>\nu(N)$, then the group will be distributed a total amount which is larger than what is at their disposal (Vorob'ev, 1977).

Optimum solutions in cooperative games are obtained by finding the best set of imputations for the game. There are many methods that are available to accomplish this such as obtaining the core and stable sets (c.f. Petrosjan and Zenkevich, 1996). To calculate a fair allocation of the total gain to the players, we will use the Shapley values, which are well known to have attractive properties of a fair allocation (Shapley, 1953). The Shapley value to Player $i \in N$ under the characteristic function $\nu($.$) is given by$

$$
x_{i}=\sum_{K \subseteq N-\{i\}} \frac{|K| !(|N|-|K|-1) !}{|N| !}(\nu(K \cup\{i\})-\nu(K))
$$

where $|K|$ refers to the cardinality of the coalition $K$ and the summation is over all coalitions $K$ excluding $P_{i}$.

\subsection{The characteristic function of the phosphorus pollution game}

In this section, we formulate the characteristic function for our phosphorus pollution game. This characteristic function incorporates both the NEP and Pareto equilibria discussed earlier in Section 1. First of all, recall that the payoff for the simplified game is

$$
\begin{aligned}
u_{i}(s)=\sum_{r=1}^{R}\left[p_{r} Q^{r} A_{i}^{r}\left[\alpha_{i}^{r} q_{i}^{r}+\alpha_{i}^{0}\right]^{\gamma}\left(W_{i}^{r}\right)^{1-\gamma}-F A_{i}^{r} \alpha_{i}^{r}\right. \\
\\
\left.\quad-\sum_{j=1}^{N} \beta_{i j} E A_{j}^{r}\left(\alpha_{j}^{r}\left(1-q_{j}^{r}\right)-L\right) I\left(\alpha_{j}^{r}\left(1-q_{j}^{r}\right)>L\right)\right]
\end{aligned}
$$

where

$$
\begin{aligned}
\gamma= & \text { Cobb-Douglas constant } \\
q_{i}^{r}\left(\alpha_{i}^{r}, t_{i}^{r}\right)= & \text { proportion of phosphorus that is released into farmland devoted to crop } r ; \\
1-q_{i}^{r}\left(\alpha_{i}^{r}, t_{i}^{r}\right)= & \text { proportion of phosphorus that flow into the effluent river systems as a } \\
& \text { consequence thereof; } \\
E\left(t_{i}^{r}\right)= & \text { environmental impact manifested as cost per unit application of } \\
& \text { phosphorus; } \\
A_{i}^{r}= & \text { total quantity of land devoted to crop } r \text { by } P_{i} ; \\
W_{i}^{r}\left(t_{i}^{r}\right)= & \text { amount of water available at time } t_{i}^{r} ; \\
Q^{r}\left(t_{i}^{r}\right)= & \text { quantity of crop } r \text { produced per unit area per unit phosphorus per unit of } \\
& \text { water }{ }^{1-\gamma} ; \\
p_{r}= & \text { price (revenue) obtained per unit of crop } r \text { sold; } \\
\alpha_{i}^{0}= & \text { base quantity of phosphorus in soil of user } i \text { per unit area; } \\
F= & \text { price per unit of phosphorus fertiliser } ; \\
\beta_{i j}= & \text { environmental influence indirectly induced on } P_{i} \text { by } P_{j} \\
L= & \text { toxicity threshold level, i.e. the amount of phosphorus in the effluent river } \\
\text { and } & \text { systems above which there will be a negative environmental impact }
\end{aligned}
$$

and the Pareto objective function is

$$
Z(s)=\sum_{i=1}^{n} w_{i} u_{i}(s)
$$

where $s=\left(\alpha_{1}, \alpha_{2}, \ldots, \alpha_{n}\right)$ and $w_{i}>0 \forall i, \sum_{i=1}^{n} w_{i}=1$. The Nash solution $\hat{s}_{N}=\left(\alpha_{1}, \alpha_{2}, \ldots, \alpha_{n}\right)$ for crop $r$ is the value that optimizes $u_{i}(s)$ for all $i$ and is given by equation (9) in Schreider et al. (2010). 
The Pareto solution $\hat{s}_{P}=\left(\alpha_{1}, \alpha_{2}, \ldots, \alpha_{n}\right)$ for crop $r$ is the value that optimizes $Z(s)$ and is given by equation (10) (ibid). (Note that we can ignore the $w_{i}^{\prime} s$ in this equation if $w_{i}=\frac{1}{n} \forall i$ and this is what will be assumed here.)

Consider the coalition

$$
=\left\{i_{1}, i_{2}, \ldots, i_{r}\right\}
$$

and let the notation $\alpha_{K}$ stand for

$$
\alpha_{K}=\left(\alpha_{i_{1}}, \alpha_{i_{2}}, \ldots, \alpha_{i_{r}}\right) .
$$

Define the vector

$$
\hat{\alpha}_{K, P}=\left(\hat{\alpha}_{i_{1}}, \hat{\alpha}_{i_{2}}, \ldots, \hat{\alpha}_{i_{r}}\right)
$$

where $\hat{\alpha}_{i_{j}}$ is given by (9) from Schreider et al. (2010) and the vector

$$
\hat{\alpha}_{K^{c}, N}=\left(\hat{\alpha}_{j_{1}}, \hat{\alpha}_{j_{2}}, \ldots, \hat{\alpha}_{j_{n-r}}\right)
$$

where $\hat{\alpha}_{j_{k}}$ is given by (10) from Schreider et al. (2010) and

$$
K^{c}=\left\{j_{1}, j_{2}, \ldots, i_{n-r}\right\}
$$

is the counter-coalition to $K$. Define the set function $\nu():. 2^{N} \longmapsto R$ by

$$
\nu(K)=\sum_{i \in K} u_{i}\left(\hat{\alpha}_{K, P}, \hat{\alpha}_{K^{c}, N}\right)
$$

Proposition. The function $\nu($.$) is a characteristic function, i.e. it satisfies the following two conditions:$

1. $\nu(\emptyset)=0$.

2. $\nu(A \cup B) \geq \nu(A)+\nu(B)$ for any mutually exclusive sets $A$ and $B$.

Condition 1 is easily seen to be satisfied by the definition of $\nu($.$) . Let A=\left\{i_{1}, i_{2}, \ldots, i_{m}\right\}$ and $B=$ $\left\{j_{1}, j_{2}, \ldots, j_{n}\right\}$ where $A \cap B=\emptyset$; to prove that the second assumption holds, we are required to show

$$
\begin{aligned}
\sum_{i \in A \cup B} u_{i}\left(\hat{\alpha}_{A \cup B, P}, \hat{\alpha}_{(A \cup B)^{c}, N}\right) & \geq \sum_{i \in A} u_{i}\left(\hat{\alpha}_{A, P}, \hat{\alpha}_{A^{c}, N}\right) \\
& +\sum_{i \in B} u_{i}\left(\hat{\alpha}_{B, P}, \hat{\alpha}_{B^{c}, N}\right) .
\end{aligned}
$$

Since $Z\left(\hat{\alpha}_{K, P}, \alpha_{K^{c}}\right)=\max _{\alpha_{K}} Z\left(\alpha_{K}, \alpha_{K^{c}}\right)$ it follows that

$$
\begin{aligned}
\sum_{i \in A \cup B} w_{i} u_{i}\left(\hat{\alpha}_{A \cup B, P}, \hat{\alpha}_{(A \cup B)^{c}, N}\right)= & \max _{\alpha_{A \cup B}} \sum_{i \in A \cup B} w_{i} u_{i}\left(\alpha_{A \cup B}, \hat{\alpha}_{(A \cup B)^{c}, N}\right) \\
= & \max _{\alpha_{A \cup B}} \sum_{i \in A} w_{i} u_{i}\left(\alpha_{A \cup B}, \hat{\alpha}_{(A \cup B)^{c}, N}\right) \\
& +\max _{\alpha_{A \cup B}} \sum_{i \in B} w_{i} u_{i}\left(\alpha_{A \cup B}, \hat{\alpha}_{(A \cup B)^{c}, N}\right) \\
\geq & \max _{\alpha_{A}} \sum_{i \in A} w_{i} u_{i}\left(\alpha_{A}, \hat{\alpha}_{A^{c}, N}\right) \\
& +\max _{\alpha_{B}} \sum_{i \in B} w_{i} u_{i}\left(\alpha_{B}, \hat{\alpha}_{B^{c}, N}\right) \\
= & \sum_{i \in A} w_{i} u_{i}\left(\hat{\alpha}_{A, P}, \hat{\alpha}_{A^{c}, N}\right) \\
& +\sum_{i \in B} w_{i} u_{i}\left(\hat{\alpha}_{B, P}, \hat{\alpha}_{B^{c}, N}\right) .
\end{aligned}
$$

Finally, setting $w_{i}=\frac{1}{N} \forall i=1,2, \ldots, N$ in both sides of equation (14) and canceling completes the proof of the proposition. 


\section{Application of Coalition game-theoretic model to the Hopkins River Case STUDY}

In this section, we outline our approach to the computation of Shapley values for farmers in the Hopkins River catchment area. Deng and Papadimitriou (1994) have shown that the task of computing Shapley values is an NP-complete problem. To obtain the Shapley values defined by (12) for $n=30$ players involves calculating $2^{30}-1$ different characteristic function values corresponding to the total number of possible coalitions which contain players in them. This is a gargantuan task due to the restriction on the number of variables and vectors allowable in programming software tools such as MATLAB. Even if this was possible, running a program containing such an enormous number of variables would utilize a huge amount of CPU running time. Our approach in calculating Shapely values is to calculate each characteristic function first and then update the Shapely values for the 30 players. Using this approach we don't have to save the characteristic functions and overwrite them after updating each Shapley value. Therefore, we just have to deal with a single characteristic function each time. For example, when $K=$ $\{1,2,3\}, \nu(K)$ is calculated and if $i$ is in $K$, it is multiplied by $(|K-1| !(N-|K-1|-1) !) / N !$ and then added to $x_{i}$, the Shapley value for $P_{i}, i \in K$; if $i \ni K$, it is multiplied by $(|K| !(N-|K|-1) !) / N$ ! and is deleted from $x_{i}$. The Pseudocode of the steps outlined are as follows:

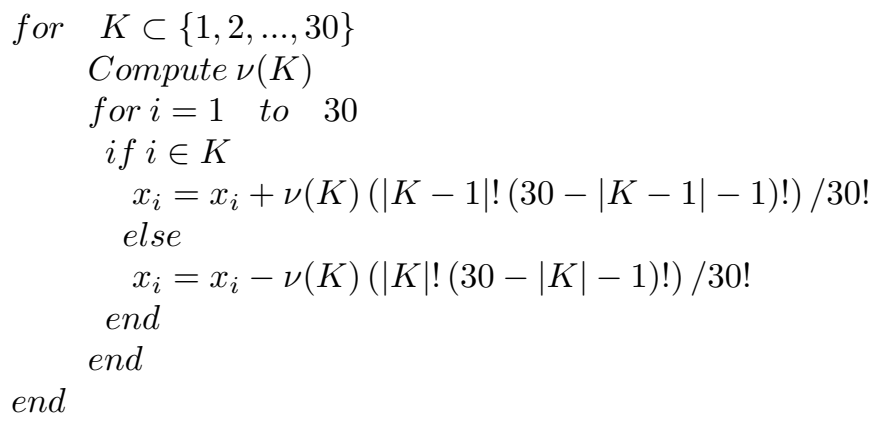

The program used to calculate Shapley values is implemented in MATLAB 7.1 on a PC equipped with INTEL 2 Core, 1.83- GHz CPU and 512 MB RAM memory. The program took about 603700 seconds (7 days) to run. The Nash and Pareto payoffs for all players based on the Nash equilibrium, Pareto optimum solutions and Shapley values are displayed in Table 1. Although the results are very similar for most players, it is clear from the fifth column that only $P_{21}, P_{23}$ and $P_{26}$ will obtain less utility under the Pareto solution than a Nash one. The last column shows that the imputations obtained by the players from their Shapley values when they cooperate all exceed their corresponding Nash values. Both of these results provide some consensus to the proposition that adopting a cooperative approach (Pareto and Shapley) will yield more revenues for the players than a non-cooperative approach (Nash).

\section{IS COOPERATION A REALISTIC OPTION?}

In the present study, the objective of each agent is to increase its own revenue, this approach means that all farmers (or groups of farmers) are posited in a competitive framework. However, cooperation sometimes plays a more significant role in resource management than pure competition because the vast majority of community members share the same ideals about fairness and environmental values such as water quality, soil salinity, biodiversity etc. The current research employs the theory of cooperative games as an alternative to this purely competitive approach. The major problem of all game theoretic models based on cooperation is that for some players or coalition of players their individual non-cooperative behavior leaves them better off than to cooperate. Disregarding to the fact that the collective system could loose it makes some players tempted to cheat. The major problem of all cooperation approaches is how realistic it is to avoid such cheating. Firstly, the significant feature of this research work, allowing us to overcome this problem, is that it has been organized with close stakeholder participation during all stages of the project implementation. This participation has two basic components. Firstly, farmers were given the opportunities to participate in formulating of the cooperative doctrine of the game and, secondly, they discussed adoption of the project's results in their routine farm management work. The stakeholder participation was implemented through the communication with different community networks in the Hopkins River catchment (Schlapp and Schreider, 2007). These community groups unify farmers by their professional interest or by spatial proximity of their estates. The research team communicated with 
Table 1: Comparisons between Nash, Pareto and Shapley utilities

\begin{tabular}{|c|c|c|c|c|c|}
\hline Player & Nash & Pareto & Shapley values & Pareto - Nash & Shapley value - Nash \\
\hline 1 & 163895.6 & 163899.2 & 163897.8 & 3.527804 & 2.187537 \\
2 & 135741.2 & 135742.5 & 135741.7 & 1.329903 & 0.58486 \\
3 & 270643 & 270643.1 & 270643.1 & 0.110645 & 0.072512 \\
4 & 333118.6 & 333118.6 & 333118.6 & 0.079465 & 0.04764 \\
5 & 235766.7 & 235766.7 & 235766.7 & 0.046963 & 0.021362 \\
6 & 408301.2 & 408301.3 & 408301.2 & 0.087921 & 0.059747 \\
7 & 77257.31 & 77257.42 & 77257.36 & 0.115191 & 0.049906 \\
8 & 26.03786 & 26.04052 & 26.03875 & 0.002666 & 0.000891 \\
9 & 221029 & 221029.2 & 221029.1 & 0.182532 & 0.088136 \\
10 & 15701.15 & 15701.2 & 15701.17 & 0.045494 & 0.015311 \\
11 & 234249.7 & 234249.8 & 234249.8 & 0.066908 & 0.035793 \\
12 & 101471.5 & 101471.6 & 101471.6 & 0.110975 & 0.042742 \\
13 & 44431.55 & 44431.62 & 44431.57 & 0.076879 & 0.026302 \\
14 & 357260.6 & 357260.7 & 357260.7 & 0.074934 & 0.097201 \\
15 & 14256.42 & 14256.7 & 14256.52 & 0.274629 & 0.09291 \\
16 & 118973.8 & 118975.1 & 118974.3 & 1.33212 & 0.570237 \\
17 & 738285.4 & 738285.4 & 738286 & 0.066084 & 0.609877 \\
18 & 154279.4 & 154280.9 & 154280.6 & 1.4718 & 1.247507 \\
19 & 59448.25 & 59451.32 & 59449.74 & 3.066866 & 1.485655 \\
20 & 148713.8 & 148715.2 & 148715.1 & 1.420105 & 1.295108 \\
21 & 674213.6 & 674211.9 & 674218.1 & -1.70561 & 4.554878 \\
22 & 170434.4 & 170436 & 170435.2 & 1.641164 & 0.809804 \\
23 & 395551.7 & 395550.6 & 395553.2 & -1.09508 & 1.52967 \\
24 & 9723.286 & 9727.513 & 9724.648 & 4.226424 & 1.361566 \\
25 & 222732.9 & 222737.1 & 222735.6 & 4.213054 & 2.780739 \\
26 & 231674.7 & 231671.9 & 231679.2 & -2.81854 & 4.4448 \\
27 & 25.49782 & 31.75691 & 27.51986 & 6.259093 & 2.022039 \\
28 & 443.6113 & 448.7244 & 445.262 & 5.113098 & 1.65066 \\
29 & 467025.3 & 467027.4 & 467029.4 & 2.125083 & 4.10194 \\
30 & 108980.6 & 108981.9 & 108981.4 & 1.29152 & 0.852762 \\
\hline & & & & & \\
\hline
\end{tabular}

established farmer groups in south west Victoria to obtain information on the fertilizer practices on individual properties within the Hopkins River basin and to communicate the model implementation results with the farmers' community. The community groups 'Southern Farming Systems', Glenthompson Bestwool/Bestlamb Group, Muston's Creek Landcare Group, Bushy Creek Group and the South Eckland Dairy Discussion Group were approached. The role of these community groups is crucially important for maintaining cooperation. In these groups farmers share their experience in their managerial practices and mutually develop new strategies of developming their farms. These community groups make the farming technologies used in practice very transparent and almost exclude opportunities for cheating the partners. The close link of research context with these farmer's community groups, which were the major lever of project results delivery, is the main reason which makes cooperation a stable strategy and eliminates the temptation to cheat. The second main reason which hindered farmers to choose a cheating strategy is that farming is a business with relatively long time horizons. It means that the benefit from selecting a cheating strategy in a particular year is marginally small compare to the prospect to damage the reputation of honest player which is so important in long run. However, farmers usually built their strategies on longterm expectations because of very high value of transition costs in agricultural business. It makes an assumption of 'no cheating' behavior of players from the game theoretic model constructed in the present research, quite plausible.

\section{Conclusions}

This paper describes the application of cooperative game theoretic approach to the problem of effective management of phosphorus fertilizers in order to minimize the environmental impact to waterways. This problem was treated as tradeoff between farmers intention to maximize their crop revenue trying the same time to keep environment healthy. The reported results indicate that the cooperative approach has a clear advantage over the competitive strategies. The results of farmers' survey indicated a good knowledge of the agricultural practices which contribute to nutrient pollution. They are willing to be a part of the process of reducing possible nutrient runoff as they can see the benefit to production, however few regarded nutrient runoff as a current problem in their own catchment. By raising awareness of the actual status of the region's rivers and the potential impact of land use practices will highlight the off-site benefits. The major message of this research sent to farmers is the amount of fertilizers, expressed in tons of phosphorus per hectare, which farmers should use in their property in order to have 'optimal' outcome. In the 
non-cooperative game framework this 'optimality' can be obtained following strategy corresponding to the unique Nash equilibrium or to one of many 'non-dominated' Pareto optima. The first approach corresponds to the competitive framework whereas the second one corresponds to some form of partnership cooperation. As evident from the objective function values displayed in Table 1, the cooperative strategies usually lead to better outcomes for farmers than competition. However two problems appear as evident from Table 1: firstly, Pareto optima do not always dominate Nash optima. Secondly, the non-uniqueness of Pareto solutions makes it difficult for players to select the most appropriate ones. This leads to some ambiguity in strategy selections. The result of this research however, lends some support to the notion that farmers' cooperation via the formation of coalitions is perhaps the best action to take. It should be clearly stated that Shapley values do not provide the alternative solution for the game but just demonstrate the existence of total income redistribution which always dominates over the Nash equilibrium values of objective function. This income distribution can very likely be unstable, in sense that for some players or coalitions of players some strategy can lead to better outcomes, than that suggested by Shapley value approach. This situation becomes more likely with increasing the number of players. In other words, the core of the game is most likely empty in a game with a large number of players. The case of the games with non-empty cores is considered by Gurvich and Schreider (2011) when players are situated on the non-cyclic graphs, like for instance in the gravity delivered irrigation systems. The total payoff of a cooperative approach, i.e. adopting the Pareto method, is greater than that using Nash equilibrium. This should encourage farmers to establish a coalition in order to optimize their gains cooperatively. Since using individual payoff of "non-dominated" Pareto optima doesn't always comply with individual rationality as can be seen from the results in Table 1 (c.f. 5th column), players 21, 23 and 26 may prefer to proceed with their individual non-cooperative objective (Nash equilibrium). However, using Shapley values guarantees that none of the farmers would earn less utility when compared to Nash equilibrium strategy (c.f. 6th column in the Table 1).

\section{ACKNOWLEDGMENT}

We thank Matthew Fernandes, who participated in development of 'competitive' version of the game theoretic model. We acknowledge Julia Schlapp for designing and implementing the household survey in the Hopkins River catchment. We are especially grateful to Professor Vera Bergelson from Rutgers University, for many challenging discussions on cooperative behaviour.

\section{REFERENCES}

Deng, X. and Papadimitriou, C.H. (1994), On the complexity of cooperative solution concepts, Mathematics of Operations Research, 19 (2), pp. 257-266.

Dinar, A. (2004). Cooperation in managing trans boundary water resources: evaluation, approaches and experiences. Proceedings of 4th Rosenberg International Forum on Water Policy,

Filar, J.A. and Gaertner, P.S. (1997). A regional allocation of world $\mathrm{CO}_{2}$ emission reductions. Mathematics and Computers in Simulation, 43, pp. 269-275.

Gurvich, V. and Schreider, S. (2011). Stable families of coalitions for network resource allocation problems.Contribution to Game Theory and Management. (IV), Petrosjan, L.A. and Zenkevich, N.A. (editors), Graduate School of Management, St. Petersburg University (in press).

Petrosjan, L. and Zenkevich, N. (1996). Game Theory. (Series on Optimization Vol. 3). World Scientific, Singapore, New jersey, London, Hongkong.

Schlapp, J. and Schreider, S. (2007). Survey of farmer management of phosphorus application in the Hopkins River catchment, for use in game-theoretic modeling. MODSIM 2007 International Congress on Modelling and Simulation, pp. 2361-2367.

Schreider, S., Zeephongsekul, P. and Fernandes, M. (2010). Modelling the optimal strategies of fertiliser application using the game-theoretic approach, Environmental Modeling and Assessment, 15(4), pp. 223-238.

Shapley, L.S. (1953). A value for n-person games. In Contributions to the Theory of Games, vol. II, Kunh, H. and Tucker, A. (editors). Princeton Univ. Press, Princeton.

Vorob'ev, N.N. (1977). Game Theory: Lectures for Economists and Systems Scientists. Springer, New York. 